\title{
10
}

\section{TRANSFORMING ASTROPHYSICS IN A PLANETARIUM: 'WE ARE PART OF THE UNIVERSE, THE UNIVERSE IS PART OF US'}

\author{
Line Bruun Nicolaisen, Marianne Achiam, and Tina Ibsen
}

Knowledge of astronomy has always held power. From ancient times, there has been a divide between those close to the sky, and those far from it; a divide of often-divine connotations (Selin, 2000). This uneven distribution of power has persisted from early astronomy to its modern Western forms (Gorman, 2005; Penprase, 2011). Today, inequitable power structures are formed throughout scientific culture (e.g., Haraway, 1988; Harding, 1991).

When museums create exhibitions about science, they purposefully deconstruct scientific knowledge, values and practices and reconstruct them to create environments that appeal to their visitors. This de- and reconstruction runs the risk of inadvertently reproducing the biases, dilemmas and inequalities of science (cf. Levin, 2010; Nicolaisen \& Achiam, 2020). Ultimately, the inequalities reproduced in this way can lead to a misalignment between the institutions and those they are set to serve, which results in social, economic and ethnic exclusion (cf. Dawson, 2014). This problem has been observed and challenged more recently across a range of museums (e.g., Christensen, 2016; Robinson, 2017).

Uncovering, analysing and critiquing these biases are pursuits in their own right. Here, however, we discuss how to go even further by promoting change. We are aligned with 'third wave' museology, which focusses on both exhibition design and practice (Macdonald, 2007; Macdonald \& Basu, 2007) when addressing the dilemmas museums are facing (Hein, 2010). We report on the first phase of a study which builds on theory through design (Markussen, 2017) by linking research, design and practice through an iterative design experiment as a means to address gender inequity. Specifically, we discuss how the Planetarium in Denmark underwent a redefinition process, emerging as an example for institutions facing similar dilemmas (cf. Hein, 2010). 
In general, planetariums differ from museums in that they do not rely on objects from collections to disseminate their scientific and technical subject matter. However, like museums they disseminate scientific subject matter to the general public, and like some science centres and museums, the Planetarium has been challenged by dwindling visitor numbers in recent years. In response, it launched a strategy in 2016 to re-establish its relevance as an out-of-school science education provider. This included inviting a science education researcher to participate in the development of the new permanent exhibition Made in Space. The aim of this collaboration was to create an exhibition design that balanced the requirement of representing authentic astrophysics, space technology and planetary science (ASTPS) with the need to create inclusive experiences.

\section{Existing research}

The objective of Made in Space was to make recent research on ASTPS inclusive, relevant and available for a diverse audience. This task is challenging, given the uneven distribution of power and access to ASTPS (Griffin, 2014). The uneven distribution is manifest, for instance, in the Space Race Model (Gorman, 2005), which presents space discovery as a triumph of technological achievement and the natural human urge to explore. It thereby creates a master narrative of the interests of largely white male American astronauts, space administrators, scientists and politicians as universal human values, and downplays the military, nuclear, nationalist and colonial aspirations of space faring nations (Bryld \& Lykke, 2000). This master narrative leaves little room for other perspectives, effectively 'devaluing previous, non-technological or other connections to space' (Griffin, 2014, p. 39).

Evidence of these other perspectives is scarce, as they are found in different contexts and manifest themselves in different ways. Recent studies point out how women's voices are systematically excluded from astronomy (Caplar et al., 2017), how the views of Indigenous people in Hawai'i on astronomy are ignored (Ciotti, 2010) and how Indigenous people in Australia must compete with the needs of a rocket range for water (Gorman, 2007). We argue that these transgressions result from a broader 'culture of positivism' that positions an existing body of knowledge as neutral and scientific rather than as a tool to serve certain groups (Kincheloe \& Tobin, 2009). This culture leads to exclusionary practises within both research and research communication. We thus urge the community of ASTPS researchers and practitioners to act (cf. Johnston, 2019); we ourselves take action by asking: How can the inequalities of ASTPS be purposefully replaced with more equitable and inclusive framings of knowledge in the development of an exhibition? Based on a brief theoretical outline and an overview of dominant ASTPS discourses, our answers in the following are based on the authors' co-design of and research on the initial stages of developing the Made in Space exhibition. We discuss these answers with a view to future developments in experimental museology. 


\section{Theoretical framework}

Our research acknowledges that people's ideas, values and knowledge are shaped by social, political, cultural, economic, gender and ethnic experiences. Science is not exempt from those influences. As discussed, we are interested in how the inequalities inherent in interactions with ASTPS may epistemologically empower or disempower certain groups or individuals. Consequently, we locate this research in the critical theory research paradigm (Treagust et al., 2014), which just like critical museology (Shelton, 2013) stems from the Frankfurt School (Kincheloe \& McLaren, 2005). Both have a focus on practice; however, as their domains differ, they tackle different dilemmas and problems in their efforts to challenge and transform institutions for the betterment of the people involved (Treagust et al., 2014).

We see institutions as being defined by the rules, conventions and indeed power dynamics that constrain and enable people's behaviour and thereby structure social interactions. These rules and conventions may be explicit and accepted as official, or they may be implicit, created and enforced outside officially sanctioned channels (Waylen, 2014). Institutions, in turn, are embedded in broader societal and cultural contexts with their own sets of rules and conventions that interact with those of the institutions. This view of institutions as being embedded in societies, and knowledge being embedded in institutions, is reflected in our choice of the anthropological theory of didactics (ATD) as the framing for this study (Bosch \& Gascón, 2014). The theoretical backdrop of ATD is inspired by the French sociologists Marcel Mauss and Michel Verret; in addition to emphasising the nested nature of knowledge, the theory acknowledges the fundamental human nature of all scientific knowledge (Chevallard \& Bosch, 2014). Thus, knowledge is always shaped by the (societal, institutional, disciplinary) ecology that it 'lives' within; when knowledge is transplanted to a new context, it is necessarily reshaped to that particular ecology.

Accordingly, we conceptualise the development of Made in Space as the selection of ASTPS-related knowledge, values and practices, produced within and adapted to research institutions, and the subsequent deconstruction and reconstruction of the selected ASTPS-related knowledge, values and practices with the purpose of making them viable in a cultural institution, namely a planetarium (cf. Mortensen, 2010). This study thus tracks the changes in the ASTPS-related knowledge, values and practices through their gradual transformation from research context to dissemination context. Of particular importance are the ways in which the inequalities of ASTPS are purposefully replaced with more equitable and inclusive framings of knowledge. In the following, we describe the inequalities of ASTPS in terms of dominant discourses, which we see as elements of the 'culture of positivism' of the ASTPS research disciplines.

\section{ASTPS as masculine-gendered}

A growing body of research provides evidence of the masculine gendering of astrophysics and related sciences. From the inception of space exploration, masculine 
adventure has been a constant referent (Redfield, 2002), most recently embodied by the postponement of the world's first all-female spacewalk due to the unavailability of space suits in fitting sizes (Connley, 2019). However, this gender inequality is also present in more academic aspects of ASTPS, e.g., citation counts in astronomy publications (Caplar et al., 2017) or workplace experiences (Clancy et al., 2017).

\section{Space research for the good of mankind}

Griffin (2014) describes how space industry is often discussed in terms of providing humanity with universal benefits, e.g., ensuring a skilled workforce for the future or educating the global public about the importance of space. Often, Griffin writes, these discussions seem to lack an awareness of people who are not part of the logic of space technology (e.g., by being employed or otherwise occupied by it). Related to the notion of benefit to mankind is the perception of ASTPS research as a selfless response to a higher calling (Redfield, 2002), or even 'sacred duty' (Whitten, 1996).

\section{Exploration-colonisation terminology}

The link between space travel and colonial history is familiar to most. Research describes how in discussions of space travel, frontier metaphors and nationbuilding vocabulary habitually invoke Columbus (Pecker, 1987) as well as terms such as 'conquest,' 'settlement,' 'expansion' and even 'colony' in spite of their present-day negative connotations (Redfield, 2002).

\section{Epistemic authority}

Hilgartner (1990) describes how an important narrative among scientists is that of scientific knowledge as their exclusive preserve. Public communication, in contrast, involves the creation of simplified representations for the public to grasp. This distinction serves to ensure the primacy or epistemic authority of scientific knowledge over other kinds of knowledge and has been observed more recently as well (e.g., Davies, 2008; Garvin, 2001). It also seems to exist among astrophysicists; certainly Griffin (2014) provides evidence of how the space industry seems to place the public in an asymmetrical position of ignorance.

In sum, the four dominant discourses in ASTPS are related to masculine gendering, space research as a common good, exploration-colonisation and the epistemic authority of science. These dominant discourses comprise the analytical lens for the present study.

\section{Development of Made in Space}

The exhibition Made in Space opened at the Planetarium in February 2018. The focus here is a series of exhibition workshops that took place in 2016-2017, 
designated as the scientist workshop, the core team workshop and the design workshop. Analytically, the development had two main phases: the 'what' phase, in which ASTPS knowledge, values and practises were selected to be included in the prospective exhibition, and the 'how' phase, in which the selected content was transformed and restructured for educational purposes (Figure 10.1). The main actors involved in the development process are described in Table 10.1.

The objective of Made in Space was to present ASTPS in a way that was 'accessible, engaging and entertaining to a broad diversity of visitors' (Ibsen et al., 2017).

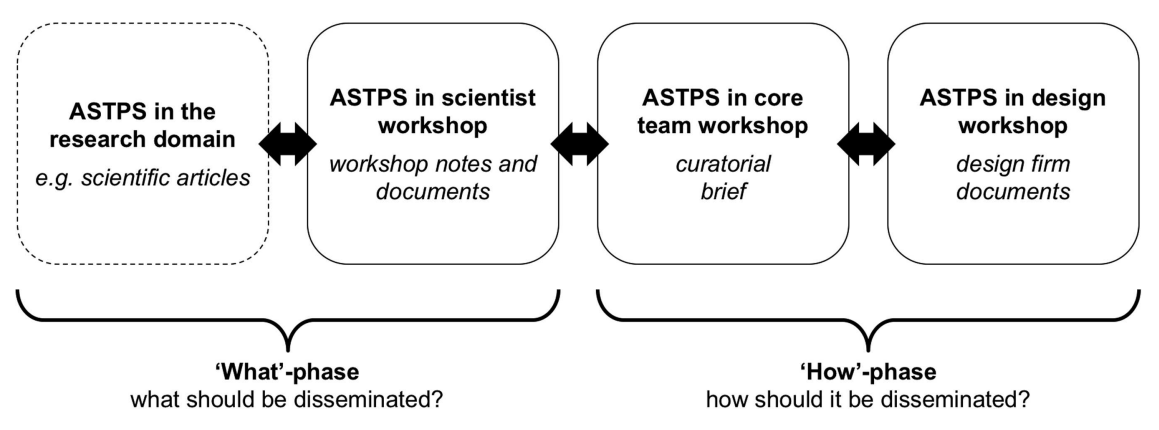

FIGURE 10.1 The development of Made in Space took place in a series of workshops and was recorded in a number of documents (shown in italics). Analytically, the development of the exhibition can be divided into a 'what'-phase and a 'how'-phase. Figure adapted from Sandholdt and Achiam (2018).

TABLE 10.1 The main actors involved in the development of Made in Space. The astrophysicists are all members of faculty at Danish universities, while the communicator and planetarium staff members have graduate degrees in astrophysics and work professionally with science communication. The researcher is a $\mathrm{PhD}$ fellow in science education, and the designers are employed at a professional exhibition design firm.

\begin{tabular}{llll}
\hline Actor & Scientist workshop & Core team workshop & Design workshop \\
\hline Astrophysicist 1 & $\mathrm{X}$ & & \\
Astrophysicist 2 & $\mathrm{X}$ & & \\
Astrophysicist 3 & $\mathrm{X}$ & & \\
Astrophysicist 4 & $\mathrm{X}$ & & \\
Astrophysicist 5 & $\mathrm{X}$ & & \\
Communicator & $\mathrm{X}$ & $\mathrm{x}$ & $\mathrm{x}$ \\
Project leader & $\mathrm{X}$ & $\mathrm{x}$ & $\mathrm{x}$ \\
Planetarium staff 1 & $\mathrm{X}$ & $\mathrm{x}$ & \\
Planetarium staff 2 & $\mathrm{X}$ & $\mathrm{x}$ & $\mathrm{x}$ \\
Planetarium staff 3 & & $\mathrm{x}$ & $\mathrm{x}$ \\
Researcher & $\mathrm{X}$ & & $\mathrm{x}$ \\
Designer 1 & & & $\mathrm{x}$ \\
Designer 2 & & & $\mathrm{x}$ \\
Designer 3 & & & \\
\hline
\end{tabular}


The core team was informed by a study of a previous Planetarium exhibition, Space Mission, which demonstrated how the masculine gendering of ASTPS found across academic contexts had been reproduced in the opportunities for interaction and meaning making in Space Mission (Nicolaisen \& Achiam, 2020). Accordingly, the development of Made in Space was intended as a means to not just produce a new, and more inclusive, exhibition, but also contribute to a general model of inclusive exhibition development for the Planetarium.

\section{Methodology}

The results presented here are based on audio recordings of the three workshops, field notes taken by the researcher (the first author), and the documents produced during the workshops and between them. The data were analysed using the six steps of thematic analysis (Braun \& Clarke, 2006), with the dominant discourses (cf. Hughes, 2001) of ASTPS as an analytical lens. We (first and second authors) coded the data independently of each other, and subsequently compared this coding to verify our categorisations. In more than $90 \%$ of cases we agreed; in those cases where we initially disagreed, we reached a common agreement by revisiting our descriptions of the four dominant discourses. This analysis allowed us to observe the presence of the dominant discourses of ASTPS in the data as well as their gradual replacement in the development of the exhibition.

\section{Analysis}

The participants in the first phase of Made in Space were invited primarily for their position at the cutting edge of astrophysics research in Denmark, and secondarily for their experience with science communication. In the scientist workshop, these professionals were tasked with identifying the most important astrophysics research in Denmark. They were urged to focus on what this research was, not on how to disseminate it. This work resulted in a preliminary list of more than 20 topics. Subsequently, they were asked to prioritise their list to identify the three most important topics, and selected exoplanets, cosmology and black holes.

In their discussions about these topics, the participants in the scientist workshop used a number of different rationales and arguments. Unsurprisingly, one argument was of the status of these topics at the cutting edge of Danish astrophysics research, but the prevalent lines of reasoning for including these topics seemed related to the dominant discourses of ASTPS. For instance, while research on exoplanets is significant in terms of being on the cutting edge of astrophysics, the participants in the scientist workshop referred repeatedly to exploration and colonisation in the discussions, for instance: '[Exoplanets as a topic] is good, because it is "modern Columbus", it is the discovery journeys, it is things you can relate to ... you can arrive at Proxima B.' This statement explicitly invokes Columbus as well as the notion that humans may someday arrive at Proxima B, the closest known exoplanet to the Earth. It seems to reflect a frontierist ideal in which new 
territories are perceived as unowned and 'for the taking' (Small, 2017). In another example, Astrophysicist 3 discusses exoplanets, invoking a colonialist rationale as well as the idea that space research is for the good of mankind:

We do [research] because of the science, there is not necessarily any financial value in it. But I sometimes like to say that we are also doing this because we would like to find a place we can colonise someday.

It is noteworthy that their references to colonisation seem unproblematic to the participants, given the recent attention to decolonising not just museums (e.g., Robinson, 2017) but also university curricula (e.g., Andrews, 2019; Conana et al., 2016).

When Astrophysicist 3 invokes her/his motivation as scientific, not financial, in the quote shown in the preceding, she/he hints at the 'for the good of mankind' discourse of ASTPS. We found a number of references to this discourse in the participants' discussions about cosmology. One instance was observed in participants' ambition to recruit young visitors to an astrophysics study programme or career pathway, as exemplified in the following:

\begin{tabular}{ll} 
Astrophysicist 4 & $\begin{array}{l}\text { What do visitors gain from [cosmology]? } \\
\text { Astrophysicist } 2\end{array}$ \\
$\begin{array}{l}\text {.. enlightenment, on different levels, both for adults but especially } \\
\text { for children, right? What is it called - when something leads to - } \\
\text { recruitment, is that correct? }\end{array}$ \\
Astrophysicist $1 \quad \begin{array}{l}\text {.. that research is not just for the chosen few. I mean that if they } \\
\text { experience that, they might see themselves as someone who could } \\
\text { work with this. [The] recruitment thing is also why I'm here. }\end{array}$ \\
\hline
\end{tabular}

Another variation of the 'for the good of mankind' rationale is the idea of ASTPS research as a selfless response to a higher calling (Redfield, 2002). We observed this rationale numerous times in the scientist workshop, for instance: 'All science is about "what is the meaning of life and why are we here"; that is what drives every scientist' (Astrophysicist 4). At times, the participants in the scientist workshop seemed to adhere to the epistemic authority discourse. In other words, they considered ASTPS in an idealised way, giving authority to those who know and seeing the public as deficient with respect to ASTPS. This position is evident in the following:

Astrophysicist 4

Planetarium staff 1
Most people on the street, they have a worldview which is on a level with what they had in the middle ages, right? I mean, they are not at all ...

$\ldots$ yeah, and there are many people who have heard that the universe started with the Big Bang. [...] There are really many people who think that this was in one place in the Universe, and something the size of a pea exploded and then it became the whole Universe [sighs]. 
Another manifestation of this discourse was observed in participants' discussions of the capability of the general public to understand scientific knowledge production. Specifically, the participants seemed reluctant to entrust the public with aspects of science that could be misconstrued, e.g., the tentativeness of scientific knowledge, as shown in the following:

Communicator

Astrophysicist 1

Communicator
We also have to include the angle that the Big Bang model is solid.

This is a recommendation that we give the designers, that these models shouldn't be wrapped in too many reservations; that this is the best suggestion from our models. Because, I think the model that is so important to us scientists, or the theory that is completely misunderstood ... [the public seems to think that] one theory is as good as another.

It is because the word theory has two different meanings in research language and in everyday language.

Finally, we also observed references to the epistemic authority of scientific knowledge in the participants' discussions of black holes. The reference to the general public as 'mere mortals' in the second quote seems to elevate the astrophysicists to near-divine status, thereby cementing their authority:

Astrophysicist 1 A lot of people are afraid of black holes. Maybe it is also a good thing if
it is explained that it is a harmless phenomenon.
Astrophysicist 2 That is the thing that may be difficult for the... the mere mortals, right,
the thing that light is still energy, that it is the energy that is
influenced and not just... Because light has no mass, right.

In addition to these examples, we have observed a number of instances in which the workshop participants spoke more generally about the communication of ASTPS. It was interesting to note how a rule of thumb for some participants was to consider the general public in terms of a 'fourteen-year-old boy' (Planetarium staff 2, Astrophysicist 1). Based on our data, it is difficult to say what lies behind this notion, but it could be a manifestation of the masculine gendering of ASTPS observed in the research literature.

In sum, using our analytical lens of dominant discourses, we identified discourses related to 'for the good of mankind,' colonisation-exploration, the epistemic authority of scientific knowledge, and (tentatively) masculine gendering, that were part of the rationales for selecting the topics exoplanets, cosmology and black holes in the scientist workshop. These discourses served to construct an underlying 'othering,' forming a 'them' (the public) and 'us' (the experts).

The restructuring of ASTPS knowledge in the 'how' phase was shaped by careful negotiations led by the Researcher, of how to question disciplinary framings of knowledge (cf. Nicolaisen \& Achiam, 2020). The initial activity was the core team workshop (Figure 10.1), which aimed to clarify the content selected 
in the scientist workshop, and to restructure that content to reflect a core narrative for the exhibition. The product of the core team workshop was an internal document (Ibsen et al., 2017; the 'curatorial brief'), to be handed over to designers with no specialisation in ASTPS.

\section{The core team workshop}

The researcher initiated a central discussion about inclusion in the core team workshop, which focussed on shifting the emphasis of the selected content away from the restrictive, textbook-like categorisation of ASTPS observed in parts of the scientist workshop towards a more human-centred framing (cf. Hein, 2010). The following exchange exemplifies this discussion:

\begin{tabular}{|c|c|}
\hline Planetarium staff 2 & $\begin{array}{l}\text { Part of the aim is also how we use cosmology, and how we have } \\
\text { reached these understandings. How we can even study the } \\
\text { beginning ... and then it makes sense to start with us. }\end{array}$ \\
\hline Planetarium staff 3 & $\begin{array}{l}\text { If the message is that one should understand one's own place in the } \\
\text { universe, right, then it makes sense to start with ourselves. }\end{array}$ \\
\hline Planetarium staff 1 & $\begin{array}{l}\text { When it comes to cosmology, then it is the story about us. I think } \\
\text { that many [people] miss that point; they think it is something that } \\
\text { happens far away. But in the field of cosmology, it is the big } \\
\text { questions you work with. Where do we come from? What } \\
\text { processes occurred for us to be here today, and what will happen } \\
\text { in the future? }\end{array}$ \\
\hline
\end{tabular}

This discussion resulted in a restructuring of the topic cosmology. Rather than taking for granted the discipline-based chronological sequence running from the Big Bang to the present day, the participants in the core team workshop decided to take a point of departure in humans, and trace the constituent elements of humans back to their formation in the development of the universe:

Imagine that you arrive at the exhibition, and then you stand in front of something, and then it breaks you apart and says that you consist of water, and this, and this, and then it continues: 'where do these things come from?' Then you have hydrogen and helium from the Big Bang, you have the ... the small stars and so on.

(Planetarium staff 1)

Not only did the restructuring of cosmology reflect a marked departure from the primacy of scientific knowledge, it also provided a basis for a central narrative for the exhibition. Through the discussions, the exhibition's guiding question evolved into 'we are part of the universe, the universe is part of us' (Planetarium staff 1). As the workshop participants began to unfold this question, the two other selected topics (exoplanets and black holes) were fit into the larger picture: 'But in this 
way, the three topics merge into one, right? They don't have to be three different parts in the exhibition, do they?' (Project leader).

Thus, rather than having three distinct topics (exoplanets, cosmology and black holes), Made in Space now had an overarching theme based on notions from cosmology, with black holes and exoplanets as sub-themes (Figure 10.2). However, even though the restructuring of the content for Made in Space represented a deliberate departure from the epistemic authority of scientific knowledge, we still observed instances of adherence to this discourse in the core team workshop. The following example is an excerpt from a discussion about how the expansion of the universe, caused by the Big Bang, allows astronomers to effectively look 'back in time':

I think it is important that we are aware that regular people don't have that way of thinking. It shouldn't be something that we just throw at them. Because we assume that of course people know that the further you look out, the further you look back in time - but people don't know that.

(Planetarium staff 1)

Even though they echoed the astrophysicists' notions of the primacy of scientific knowledge, the participants in the core team workshop still focussed on finding ways to address the perceived knowledge gap between ASTPS experts and the public. The following exchange illustrates an aspiration to empower prospective visitors to evaluate scientific claims for themselves:

Planetarium staff 2 You can hear in the news that now the age of the universe has changed. And so people think to themselves, 'okay, so [astrophysicists] are just sitting around thinking strange thoughts' ... Planetarium staff $1 \quad$... yeah, or 'why have you invented dark matter, I don't like that.' I think it is in the way we have to present it, that we don't make science up. It is based on science, and that is how we present everything in here. If this is to be an exhibition where we also talk about how we get the results, and how we work with these things, then this will be part of it. Then we will talk about the processes and not just the final results - and then [the visitors] will also see that.

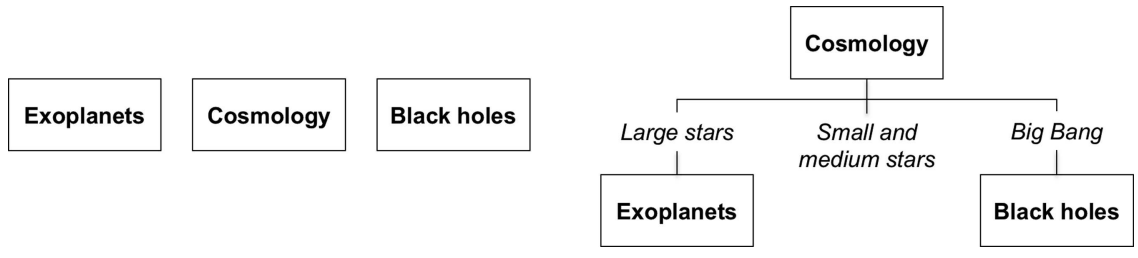

FIGURE 10.2 The restructuring of the content for Made in Space from the scientist workshop (left) to the core team workshop (right). 
The examples we observed of adherence to the primacy or authority of scientific knowledge came from workshop participants with ASTPS backgrounds. Even though these participants were employees of the Planetarium and fully invested in its raison d'être, their alliance to ASTPS disciplinary culture may have made it difficult for them to completely forego its dominant discourses (cf. Cole, 2009). Even so, the researcher's continued attention to inclusion perspectives in the design work led to a replacement of the dominant ASTPS discourses: colonisation, masculinisation of science or 'for the good of mankind' in the core team workshop. This replacement was perceived as a positive addition rather than a reduction, as suggested by the following reflection by Planetarium Staff 1:

I think it is a good thing, as well, when we talk about gender - because then we could have more people sharing one experience. Or maybe even having something that two people can interact with. That is some of the things you found as well, [Researcher], that that is more gender inclusive as well.

In summary, the participants in the core team workshop created a central narrative to encompass the three selected ASTPS topics in a coherent way and restructured those topics towards a human-centred framing likely to appeal to a broader diversity of visitors (cf. Nicolaisen \& Achiam, 2020). The dominant discourses observed in the scientist workshop were gradually replaced with more constructive considerations of the capabilities of prospective visitors, even if some members of the core team still adhered to notions of the epistemic authority of science. The results of the core team workshop were documented in the curatorial brief, which was subsequently handed over to a design firm.

\section{The design workshop}

The second activity in the 'how' phase of the development of Made in Space was the design workshop (Figure 10.1). In this workshop, the design firm presented their proposal for Made in Space, based on the curatorial brief, and discussed it with the core team. Prior to the workshop the Researcher had had extensive discussions with the design team about inclusion, and had shared a set of guidelines on key inclusion elements, including connecting scientific content with learners' bodies, the importance of social experience, emphasising cooperation rather than competition and a strong focus on visual (rather than cognitive) aspects of ASTPS (Achiam \& Holmegaard, 2017; Dancstep née Dancu \& Sindorf, 2018; Nicolaisen \& Achiam, 2020).

The design workshop marked the transposition of the ASTPS content from its written form in the curatorial brief into a different modality, namely renderings, animations and visualisations. The discussions in the design workshop were prompted by this modality shift as well as considerations of the inclusion guidelines. One prominent theme was of the intended interactions between Made in Space and its prospective visitors. In these discussions, we observed how considerations of the 
sensory and affective aspects of ASTPS partially replaced earlier considerations of its cognitive aspects. For instance, when discussing the design firm's overarching objective for Made in Space, Designer 1 focussed on creating a sense of awe and excitement among visitors, rather than 'trying to fit too much information in.' Later, as the discussion turned to concrete design ideas related to cosmology, Designer 1 again invoked the affective aspects of ASTPS:

One possibility is that we build a kind of a show moment that ties everything together. So, every thirty minutes there is one event where everybody goes 'well, this is extraordinary' [...]. So, one of the ideas is that we do the Big Bang, and that there is a single point that explodes out and kind of fills the entire space. Which would be ... which would feel amazing in that space.

Another example of the focus on sensory experiences is the designers' suggestion to use Kinect, an infrared camera system that allows the user to interface with a visualisation on a screen using their body movements, rather than buttons or toggles. Kinect supports multiple physical engagement patterns (Hsu, 2011), thus affording a diversity of ways to interact with the subject matter.

In addition to their attention to affective and sensory aspects of ASTPS, the designers also incorporated the strong focus on the social aspects of the exhibition visit as prompted by the researcher. As they were discussing the Big Bang event, Designer 2 added:

It is also about creating that communal experience. So, previously you've had lots of individual experiences, you can stand, you can do whatever you want in your space, taking your time, but then we have this moment where everyone is as one. We get this, kind of, this 'wow' moment.

Research has acknowledged the social nature of visits to cultural institutions (e.g., Falk \& Dierking, 2013). Indeed, one of the issues of the earlier Planetarium exhibition, Space Mission, was its focus on individual experiences rather than shared ones (Nicolaisen \& Achiam, 2019).

Finally, although the dominant discourses of ASTPS had been more or less completely replaced by more equitable discourses, we still observed an adherence to the primacy of scientific knowledge. The following discussion is prompted by the description of the Big Bang moment mentioned by Designer 1 in the preceding:

You show the Big Bang as one point exploding, creating the universe from one point. But that would mean that we have a centre of the universe, which we don't. [...] This is a misconception that every single science centre kind of continues to promote, and every exhibition promotes this, and people misunderstand it. So, I think that that is one of the things that we in this exhibition really need to be careful about, not to reinforce these misconceptions. 
In sum, the discussions in the design workshop were focused on the affective, sensory and social aspects of the selected ASTPS topics rather than their cognitive aspects. Although it was not always obvious in the moment that this replacement was taking place, in reflective moments, members of the team would discuss how the researcher's input had helped them change their focus: 'Yeah, I think the fact that we started this process of you talking about it as well has sort of created a different mindset' (Designer 3).

At this stage of the exhibition development, the dominant discourses observed in the scientist workshop were almost completely replaced by more equitable and inclusive constructions of ASTPS. One exception to this pattern was the lingering adherence to the epistemic authority of science.

\section{Conclusion}

In this chapter, we have documented how a practical design experiment gradually and purposefully replaced the inequalities built into ASTPS with more equitable and inclusive framings of knowledge. First, we found several dominant discourses to exist in the ASTPS of scientists and planetarium professionals, namely masculine gendering, 'for the good of mankind,' colonisation-exploration terminology and the epistemic authority of scientific knowledge. These discourses were addressed through a design experiment, based on a theoretical foundation (Macdonald, 2007; Markussen, 2017; Treagust et al., 2014) and persistent attention to the inequitable discourses. As a result, the dominant discourses were replaced by social, affective and sensory framings of ASTPS. This replacement entailed a restructuring of content as well as the transformation of its form.

We acknowledge that prospective visitors to the exhibition are not passively situated in the scientific discourses they encounter but are active agents of their own experiences (Hughes, 2001; Nicolaisen \& Achiam, 2020). This means that they bring their own perceptions and understandings to bear on Made in Space, including, potentially, perceptions of space exploration as a masculine, colonialist endeavour. However, we would argue the changes effected in the design of Made in Space allow for alternative receptions, perceptions and interaction with ASTPS content, thereby shifting the implied ways of engaging with ASTPS in a more equitable direction (Nicolaisen \& Achiam, 2020).

In this study, the 'culture of positivism' that positions ASTPS as neutral and scientific (cf. Kincheloe \& Tobin, 2009) was arguably replaced with a 'culture of relativism' in which scientific culture is not necessarily superior to the experiences and perceptions of visitors (cf. Campion, 2017). Thus, the design process can be understood as an experiment in Latour's sense of the word; 'transformative for the people and materials involved' (Latour, 1999; Macdonald \& Basu, 2007), set in a broader ecology of societal, institutional and disciplinary conditions. In the following, we discuss these ecologies in turn.

Astrophysics, space technology and planetary science are part of our shared societal and cultural ecology. ASTPS do not just belong to the élite minority of 
people directly engaged with them, but have broad significance and relevance (Gorman, 2005). For instance, it has been suggested that the most significant effect of the space programme was not its scientific or technological achievements, but ultimately, its impact on the imagination of the public (quoted in Geppert, 2018). As policy-makers and critical actors turn towards ensuring inclusive and equitable learning opportunities for all citizens (UN Sustainable Development Goal 4), more attention is being paid to exploring new ways of deconstructing science and reimagining it to create opportunities that are accessible to all members of society (De Leo-Winkler, 2019; Griffin, 2014; Johnston, 2019). It is within this wider societal ecology the Planetarium is embedded and in which the development of Made in Space represents one of an increasing number of efforts to widen public participation in ASTPS.

Of particular relevance to this discussion is institutional ecology, understood here as the symbolic meta function of science centres, museums and, we suggest, planetariums. By their symbolic function, we refer to these institutions' ability to suspend time and place to offer the visitors experiences beyond the walls and geographic location of the building (Achiam \& Sølberg, 2017). In fact, planetariums are uniquely qualified to create this suspension of time and place, given their long history of dome projection technology that allows visitors to experience 'how it is' in space (Achiam et al., 2019; Bleeker, 2017). In the present study, we saw how this institutional visual regime and imaginary (cf. Shelton, 2013) was used to reconstruct content from astrophysics, space technology and planetary science to create a proposal for an inclusive and engaging exhibition.

Finally, in developing Made in Space, the Planetarium joins the ranks of progressive science centres and museums who question authoritative, canonical science and seek instead to engage their visitors in negotiating what science means for them (Sandholdt \& Achiam, 2018). From a disciplinary point of view, this approach challenges the notion of objectivity, which has historically been an important part of the self-image of the natural science disciplines (Reiss \& Sprenger, 2017). However, a consensus is emerging that just like other processes and products of human culture, the natural sciences are constructed by and within power relations in society, not apart from them (e.g. Haraway, 1988; Harding, 1991). As a consequence, the natural sciences cannot produce culture-free knowledge (Brickhouse, 2001).

In this chapter, we have shown how a proposal for an engaging and inclusive exhibition resulted from the deconstruction and reconstruction of inaccessible and exclusive disciplinary content. We have documented how the dominant discourses of ASTPS in the initial deconstruction of the content resulted in a divide between the experts and the public, and how these discourses were disrupted through transformative experiments, which replaced the existing discourse with more inclusive ones. This deconstruction and reconstruction was explicitly guided by a critical perspective on the culture of positivism associated with the natural science disciplines (here in the form of dominant discourses of ASTPS), but as we have discussed, the particular ecology of discipline, institution and society in which the development of Made in Space was embedded played an important constructive role. 
In particular, ongoing societal discussions of inclusion, institutional considerations of planetarium modalities and the scientific disciplines' gradual coming to terms with the illusion of their objectivity created an ecological context that was conducive to the development of an engaging and inclusive proposal for an exhibition. We suggest that the development of Made in Space can be understood as a practise-based illustration of how its developers were able to constructively reconcile a number of different discourses with their actual practices.

\section{Bibliography}

Achiam, M., \& Holmegaard, H. T. (2017). Informal science education and gender inclusion. In L. S. Heuling (Ed.), Embracing the other: How the inclusive classroom brings fresh ideas to science and education (pp. 32-40). Flensburg University Press. https://www.researchgate.net/publication/ 326176462_Informal_science_education_and_gender_inclusion

Achiam, M., Nicolaisen, L. B., \& Ibsen, T. (2019). Planetariums between experience and enlightenment. Nordisk Museologi, 19(1), 11-24.

Achiam, M., \& Sølberg, J. (2017). Nine meta-functions for science museums and science centres. Museum Management and Curatorship, 32(2), 123-143.

Andrews, K. (2019). Blackness, empire and migration: How black studies transforms the curriculum. Area. Epub ahead of print. https://rgs-ibg.onlinelibrary.wiley.com/doi/full/10. 1111/area.12528?fbclid=IwAR0zu0eLTq3MUrpMR8WBsVagvuoLb3hf0qtXFwY9Vj-11_p2vflZE3ZLf8

Bleeker, M. (2017). Who knows? The universe as technospace. Early Popular Visual Culture, 15(2), 247-257.

Bosch, M., \& Gascón, J. (2014). Introduction to the Anthropological Theory of the Didactic (ATD). In A. Bikner-Ahsbahs \& S. Prediger (Eds.), Networking of theories as a research practice in mathematics education (pp. 67-83). Springer.

Braun, V., \& Clarke, V. (2006). Using thematic analysis in psychology Virginia. Qualitative Research in Psychology, 3(2), 77-101.

Brickhouse, N. W. (2001). Embodying science: A feminist perspective on learning. Journal of Research in Science Teaching, 37(5), 441-458.

Bryld, M., \& Lykke, N. (2000). Cosmodolphins: Feminist cultural studies of technology, animals and the sacred. University of Chicago Press.

Campion, N. (2017). The importance of cosmology in culture: Contexts and consequences. In A. J. Capistrano de Souza (Ed.), Trends in modern cosmology (pp. 3-17). London: IntechOpen.

Caplar, N., Tacchella, S., \& Birrer, S. (2017). Quantitative evaluation of gender bias in astronomical publications from citation counts. Nature Astronomy, 1, 0141.

Chevallard, Y., \& Bosch, M. (2014). Didactic transposition in mathematics education. In S. Lerman (Ed.), Encyclopedia of mathematics education (pp. 170-174). Springer.

Christensen, H. D. (2016). A never-ending story: The gendered art museum revisited. Museum Management and Curatorship, 31(4), 349-368.

Ciotti, J. E. (2010). Museums and planetariums: Bridging the gap between Hawaiian culture and astronomy through informal education - a case study. Forum on Public Policy, 2010(2), 1-14.

Clancy, K. B. H., Lee, K. M. N., Rodgers, E. M., \& Richey, C. (2017). Double jeopardy in astronomy and planetary science: Women of color face greater risks of gendered and racial harassment. Journal of Geophysical Research: Planets, 122(7), 1610-1623. 
Cole, K. C. (2009). Something incredibly wonderful happens: Frank Oppenheimer and the world he made up. Houghton Mifflin Harcourt.

Conana, H., Marshall, D., \& Case, J. M. (2016). Exploring pedagogical possibilities for transformative approaches to academic literacies in undergraduate physics. Critical Studies in Teaching and Learning, 4(2), 28-44.

Connley, C. (2019). NASA cancels first all-female spacewalk due to 'spacesuit availability.' CNBC Make it. www.cnbc.com

Dancstep née Dancu, T., \& Sindorf, L. (2018). Creating a female-responsive design framework for STEM exhibits. Curator: The Museum Journal, 61(3), 469-484.

Davies, S. R. (2008). Constructing communication: Talking to scientists about talking to the public. Science Communication, 29(4), 413-434.

Dawson, E. (2014). "Not designed for us": How science museums and science centers socially exclude low-Income, minority ethnic groups. Science Education, 98(6), 981-1008.

De Leo-Winkler, M. A. (2019). The universal universe or making astronomy inclusive. Nature Astronomy, 3(7), 576-577.

Falk, J. H., \& Dierking, L. D. (2013). The museum experience revisited. Left Coast Press.

Garvin, T. (2001). Analytical paradigms: The epistemological distances between scientists, policy makers, and the public. Risk Analysis, 21(3), 443-456.

Geppert, A. C. T. (2018). European astrofuturism, cosmic provincialism: Historicizing the space age. In A. C. T. Geppert (Ed.), Imagining outer space: European astroculture in the twentieth century (pp. 3-28). Palgrave Macmillan.

Gorman, A. (2005). The cultural landscape of interplanetary space. Journal of Social Archaeology, 5(1), 85-107.

Gorman, A. (2007). La Terre et l'Espace: Rockets, prisons, protests and heritage in Australia and French Guiana. Archaeologies, 3(2), 153-168.

Griffin, J. M. (2014). Experience and viewpoints in the social domain of space technology $[\mathrm{PhD}$ dissertation, University of Plymouth]. http://hdl.handle.net/10026.1/3084

Haraway, D. (1988). Situated knowledges: The science question in feminism and the privilege of partial perspective. Feminist Studies, 14(3), 575-599.

Harding, S. (1991). Whose science? Whose knowledge? Thinking from women's lives. Cornell University Press.

Hein, H. (2010). Looking at museums from a feminist perspective. In A. K. Levin (Ed.), Gender, sexuality and museums: A Routledge reader (pp. 53-64). Routledge.

Hilgartner, S. (1990). The dominant view of popularization: Conceptual problems, political uses. Social Studies of Science, 20(3), 519-539.

Hsu, H.-M. J. (2011). The potential of Kinect in education. International Journal of Information and Education Technology, 1(5), 365-370.

Hughes, G. (2001). Exploring the availability of student scientist identities within curriculum discourse: An anti-essentialist approach to gender-inclusive science. Gender and Education, 13(3), 275-290.

Ibsen, T., Rasmussen, K. S., \& Kristensen, M. T. (2017). Ny udstilling (internt dokument) [New exhibition (internal document)]. Tycho Brahe Planetarium.

Johnston, K. V. (2019). A dynamical systems description of privilege, power and leadership in academia. Nature Astronomy, 3(12), 1060-1066.

Kincheloe, J. L., \& McLaren, P. (2005). Rethinking critical theory and qualitative research. In N. K. Denzin \& Y. S. Lincoln (Eds.), Handbook of qualitative research (3rd ed.) (pp. 303-342). Sage.

Kincheloe, J. L., \& Tobin, K. (2009). The much exaggerated death of positivism. Cultural Studies of Science Education, 4, 513-528. 
Levin, A. K., (Ed.) (2010). Straight talk: Evolution exhibits and the reproduction of heterosexuality. Gender, sexuality and museums. A Routledge reader (pp. 375-394). Routledge.

Latour, B. (1999) Pandora's hope: Essays on the reality of science studies. Harvard University Press.

Macdonald, S. (2007). Interconnecting: Museum visiting and exhibition design. CoDesign: International Journal of CoCreation in Design and the Arts, 3, 149-162.

Macdonald, S., \& Basu, P. (2007). Exhibition experiments. Blackwell.

Markussen, T. (2017). Building theory through design. In L. Vaughan (Ed.), Practice based design research (pp. 87-98). Bloomsbury Academic.

Mortensen, M. F. (2010). Museographic transposition: The development of a museum exhibit on animal adaptations to darkness. Éducation \& Didactique, 4(1), 119-137.

Nicolaisen, L. B., \& Achiam, M. (2020). The implied visitor in a planetarium exhibition. Museum Management and Curatorship, 35(2), 143-159.

Pecker, J.-C. (1987). Pourquoi ce colloque? [Why this conference?]. In J. Schneider \& M. l'Eger-Orine (Eds.), Frontières et conquête spatiale: La philosophie à l'épreuve [Frontiers and space conquest: The philosopher's touchstone] (pp. 3-8). Kluwer Academic Publishers.

Penprase, B. E. (2011). The power of stars: How celestial observations have shaped civilization. Springer.

Redfield, P. (2002). The half-life of empire in outer space. Social Studies of Science, 32(5-6), 791-825.

Reiss, J., \& Sprenger, J. (2017). Scientific objectivity. In E. N. Zalta (Ed.), The Stanford encyclopedia of philosophy. https://plato.stanford.edu

Robinson, H. (2017). Is cultural democracy possible in a museum? Critical reflections on indigenous engagement in the development of the exhibition Encounters: Revealing stories of Aboriginal and Torres Strait islander objects from the British Museum. International Journal of Heritage Studies, 23(9), 860-874.

Sandholdt, C. T., \& Achiam, M. (2018). Engaging or transmitting? Health at the science centre. Nordisk Museologi: Journal of Nordic Museology, 2018(2-3), 136-151.

Selin, H. (Ed.). (2000). Astronomy across cultures: The history of non-Western astronomy. Springer. Shelton, A. (2013). Critical museology: A manifesto. Museum Worlds, 1(2), 7-23.

Small, L. M. (2017). Space museums: Technical and cultural considerations [Master's thesis, University of Toronto, Toronto].

Treagust, D. F., Won, M., \& Duit, R. (2014). Paradigms in science education research. In N. G. Lederman \& S. C. Abell (Eds.), Handbook of research on science education (vol. II, pp. 3-17). Routledge.

Waylen, G. (2014). Informal institutions, institutional change, and gender equality. Political Research Quarterly, 67(1), 212-223.

Whitten, B. L. (1996). What physics is fundamental physics? Feminist implications of physicists' debate over the superconducting supercollider. NWSA Journal, 8(2), 1-16. 